\title{
Decline in the mental health of nurses across the globe during COVID-19: A systematic review and meta-analysis
}

\author{
Abin Varghese ${ }^{1 *}$, Gigini \\ George $^{1 *}$, Sharat V Kondaguli ${ }^{1}$, \\ Abdallah Y Naser', Deepika C \\ Khakha $^{3}$, Rajni Chatterji ${ }^{4}$ \\ ${ }^{1}$ Bhopal Nursing College, Bhopal Memorial \\ Hospital and Research Centre, ICMR, \\ Ministry of Health \& Family Welfare, \\ Government of India, Bhopal, Madhya \\ Pradesh, India \\ ${ }^{2}$ Faculty of Pharmacy, Department of \\ Applied Pharmaceutical Sciences and \\ Clinical Pharmacy, Isra University, Amman, \\ Jordan \\ ${ }^{3}$ College of Nursing, All India Institute of \\ Medical Sciences, Ministry of Health and \\ Family Welfare, Government of India, New \\ Delhi, India \\ ${ }^{4}$ Department of Psychiatry, Bhopal \\ Memorial Hospital and Research Centre, \\ ICMR, Ministry of Health \& Family Welfare, \\ Government of India, Bhopal, Madhya \\ Pradesh, India \\ *Joint first authorship.
}

\section{Correspondence to:}

Mr Abin Varghese, BSN, MSc Psychiatric Nursing, PhD Scholar Faculty, Bhopal Nursing College

Bhopal Memorial Hospital and Research Centre

ICMR, Ministry of Health \&Family Welfare, Government of India Bhopal, Madhya Pradesh, India abinvarghese143@gmail.com
Background Nurses represent the major proportion of frontline health care professionals delivering 24/7 services to patients with an increased vulnerability towards COVID-19 infection. Mental health issues among nurses during the COVID-19 pandemic are poorly reported across the globe. Henceforth, a systematic review and meta-analysis was performed to explore the prevalence and determinants of mental health outcomes (anxiety, stress, depression, PTSD, insomnia) among nurses across the globe due to the COVID-19.

Methods A PRISMA compliant systematic review (PROSPERO-CRD 42020204120) was carried out to identify articles from multiple databases reporting the prevalence of mental health outcomes among nurses. Proportion random effect analysis, $I^{2}$ statistic, quality assessment, and sensitivity analysis were carried out.

Results Pooled data on mental health outcomes were generated from 25 cross-sectional studies: $32 \%$ anxiety (95\% confidence interval $(\mathrm{CI})=21 \%$ $44 \%, \mathrm{n}$ (number of studies) $=21, \mathrm{~N}$ (sample size $)=13641$ ), $40.6 \%$ stress (95\% CI $=25.4 \%-56.8 \%, \mathrm{n}=10, \mathrm{~N}=4204$ ), 32\% depression (95\% CI $=21 \%$ $44 \%, \mathrm{n}=17, \mathrm{~N}=12294), 18.6 \%$ PTSD ( $95 \% \mathrm{CI}=4.8 \%-38 \%, \mathrm{n}=3, \mathrm{~N}=638)$, $38.3 \%$ insomnia (95\% CI $=5.8 \%-78.6 \%, \mathrm{n}=2, \mathrm{~N}=261$ ) and significant risk factors for mental ailments includes; caring for COVID-19 patients, being a female, low self-efficacy, resilience, social support and having physical symptoms (sore-throat, breathlessness, cough, lethargy, myalgia, fever).

Conclusion The study results highlighted a higher proportion of poor mental health outcomes namely, anxiety, stress, depression, PTSD and insomnia among nurses from different parts of the world. Poor mental health outcomes among nurses warrants the need to implement proactive psychological interventions to deter the collapse of health care systems in responding to the pandemic and in particular all possible efforts should be undertaken to mitigate the risk factors. Health care organizations should provide support to nurses with sufficient flexibility. The disaster preparedness plan envisaged by nations should have provisions to address the mental health of nurses. Greater investment in addressing the global shortage of nurses should be given priority in national health policies. Attractive salary packages should be offered to nurses to prevent their emigration from low- and middle-income countries (LMICs).

Registration PROSPERO (CRD42020204120 
Coronavirus disease (COVID-19) or Severe Acute Respiratory Syndrome (SARS) CoV-2 has created havoc amongst people throughout the globe. Because of its greater infectivity, this pandemic has traversed various parts of the world at an unprecedented stride. The World Health Organization (WHO) has recorded more than 61.8 million cases and 1.4 million deaths as of 1st December 2020 [1]. As a result, health care facilities are overwhelmed with the patient load and strife to appease the demands of the population, placing immense strain on the frontline health care staff in exchange. Furthermore, a significant proportion of the frontline health care workers constitute nurses, providing round the clock services to patients with an increased vulnerability in getting infected [2]. Additionally, nurses' workplace environment and work conditions put them at a higher risk of getting infected by patients, which can lead to mental health problems. Previous respiratory infections of this century such as SARS, Middle East Respiratory Syndrome (MERS), Ebola have demonstrated its psychological ramifications on nurses, manifested as stress, anxiety, depression, hostility, somatization and fear [3-6]. Furthermore, a higher prevalence of mental ailments has been reported among nurses compared to physicians and other health care workers during those infections [7,8]. Likewise, COVID-19 has also impacted the psychological health of nurses which is apparent from the multiple cross-sectional studies carried out across the various parts of the globe [9-13]. Moreover, nurses from Wuhan reported severe level of anxiety and depression than other frontline health care workers during the initial phase of COVID-19 [14]. Additionally, negative health outcomes are expected to be more common among health care providers during the COVID-19 pandemic compared to previous ones (SARS and MERS), this could be attributed due to multiple factors such as the shortage of personal protective equipment in some countries, increased working load, and having unexperienced clinical staff in coping with this new clinical situation and its associated new clinical guidelines [15]. According to WHO, the COVID-19 pandemic is likely to have both long and short-term influences on mental health. Due to the importance of the mental health implications of the pandemic, WHO [16] reported a list of considerations for the mental well-being of high-risk groups especially health care providers. A previous systematic review on factors that affects the psychological well-being of health care providers has identified the following factors to be highly influencing: having appropriate training and readiness, being at higher risk of infection due to work conditions, job stress, quarantine, perceived risk, and poor organizational support [17]. Preserving both the mental state and the medical conditions of health care providers who accept significant responsibility for treating patients with coronavirus infections is critical to sustaining the quality of appropriate health care services. Therefore, it is imperative to address the psychological impact of COVID-19 on nurses as the current priority. The scrutiny of literature did not yield any systematic review or meta-analysis addressing the current review question or inclusion criteria. Moreover, WHO [18] endorses an expeditious review of pragmatic substantiation to aid policymakers to frame recommendations that may augment the optimal response of health care systems. We hypothesized that nurses would suffer from poor mental health outcomes with numerous factors influencing them. Consequently, this meta-analysis was carried out to synthesize and present current evidence as; the pooled prevalence of anxiety, stress, depression, posttraumatic stress disorder, insomnia and to explore significant factors that are associated with the onset of mental ailments among nurses working in COVID-19 and non-COVID-19 centres during this pandemic and to determine the variations in the prevalence rates for multiple mental health outcomes across different regions of the world.

\section{METHODS}

A primary search of the Joanna Briggs Institute Database of Systematic Reviews and Implementation Reports, the Cochrane Database of Systematic Reviews, PROSPERO and MEDLINE was performed before the registration of protocol to identify systematic reviews and meta-analysis that address the prevalence of mental health outcomes among nurses.

\section{Systematic review protocol registration}

The protocol for this systematic review and meta-analysis has been registered at PROSPERO International Prospective Register of Systematic Reviews-CRD42020204120.

\section{Ethical approval}

Ethical permission was not obtained for the study since we used published data that has already been ethically approved.

\section{Search process}

We conducted the systematic review and meta-analysis following the preferred reporting items for systematic review (Table S1 in the Online Supplementary Document) and meta-analysis [19]. Original articles 
published from 11/03/2020 were searched in the databases; PUBMED, Web of Science Core Collection, MEDLINE, Psych Info, Nursing and Allied Health Database, Science Direct, Corona Virus Research Database and Google Scholar. Moreover, the preprint versions published in Medrxiv and SSRN servers were also included. Furthermore, we screened the references of relevant articles to retrieve more potential articles. The final search for all databases was completed on October 5, 2020. Multiple keywords (MeSH and free text word synonyms) such as nursing staff, COVID-19, mental health, severe acute respiratory syndrome coronavirus 2, depression, anxiety, stress was used individually or in combination with Boolean operators across several databases. We also have included, a detailed search strategy (Appendix S1 in the Online Supplementary Document).

\section{Eligibility criteria}

Inclusion Criteria: Published articles that fulfilled the following criteria were included: i) Population (P): assessed nurses who were working in hospitals in any country; ii) Intervention/Exposure(I): Intervention was not applicable, but included studies that analyzed the prevalence of mental health outcomes using validated instruments due to COVID-19 pandemic; iii) Comparator/control (C): No comparator or controls were applicable as we screened for epidemiologic studies reporting prevalence; iv) Outcomes (O): Prevalence of anxiety, stress, depression, PTSD, insomnia, significant risk factors for mental ailments; and iv) published articles in English language.

Exclusion criteria: We excluded studies with the following characteristics: i) Did not give an aggregate prevalence of anxiety, stress, depression, PTSD and insomnia even after contacting the corresponding author; ii) studies reporting the combined prevalence of mental health outcomes along with other health care professionals; iii) qualitative studies, systematic review, meta-analysis, case reports, case series and non-accessible fulltext articles; and iv) studies with small sample size $(\mathrm{N}<40)$.

The titles and abstracts of studies were independently reviewed by two authors (AV, GG) for eligibility. Full texts of eligible studies were reviewed. Any disagreements between the two reviewers were solved by the third author (SV) by discussion and mutual consensus. Further RAYYAN QCRI was used to detect duplicates and search strategy management [20].

\section{Data extraction and appraisal of study quality}

The following information was extracted from all included studies: author, month and year of publication, country, socio-demographic characteristics (sample size, marital status, gender proportion), the instrument used, prevalence and significant risk factors of mental health outcomes. The methodological quality of the included studies was evaluated using the Loney criteria which is a widely used tool to assess observational studies estimating the prevalence of health-related problems [21]. This tool consists of eight items which include: (1) random sample or whole population (2) unbiased sampling frame (3) adequate sample size (4) standard measures (5) outcomes measured by unbiased assessors (6) adequate response rate and refusers described (7) confidence intervals (CI) and subgroups analysis and (8) study subjects described. Each item in the tool is assigned a score of 1 with the total score ranging from 0 to 8 , with more scores indicating a higher degree of quality.

\section{Statistical analysis}

All data were analysed using OPEN META [Analyst] software version 10.12 [22] which is an open-source cross-platform database for advanced meta-analysis (developed by the Centre for Evidence Synthesis, Brown University, School of Public Health, RI, USA) and the funnel plots were generated using COMPREHENSIVE META-ANALYSIS software version 3.0(CMA 3.0 developed by a group of experts with funding from National Institute of Health in 2006, Englewood, NJ: Biostat). In all statistical analysis, the significance level was considered at a $P$-value $<0.05$. The overall prevalence of mental health outcomes among nurses was calculated using the random-effect model according to Der Simonian and Laird's approach at 95\% confidence interval with Freeman turkey double arcsine transformation employed to stabilise the variance among studies [23,24].Heterogeneity testing was performed using the $I^{2}$ and Cochran's Q test [25].We interpreted the $I^{2}$ statistic results as follows: 0 to $40 \%$ as not important, 30 to $60 \%$ as moderate heterogeneity 50 to $90 \%$ as substantial heterogeneity, 75 to $100 \%$ as considerable heterogeneity [26].Furthermore, a leave one out sensitivity analysis and subgroup analysis were performed to address the potential sources of heterogeneity. Publication bias among the included studies was addressed by funnel plot and eggers linear regression intercept [27]. 


\section{RESULTS}

\section{Study characteristics}

The initial search across different electronic databases and grey literature yielded 1576 citations. First, a total of 371 duplicate papers were excluded, accompanied by the removal of 1149 publications from the title/abstracts screening. Among the 56 full-text articles screened, 29 were not included. After screening the full text of 56 studies, 29 were excluded based on numerous factors; non-nurses as study participants $(\mathrm{n}=3)$, the prevalence rate was not reported $(n=20)$ and the author not reachable $(n=6)$. Consequently, for further review, a total of 27 full-text articles matching the criteria for inclusion and exclusion were included (Figure 1). Among the 27 full-text articles included for systematic review, data of nurses concerning their mental health outcomes was made available to our study by requesting the corresponding author of 11 studies.

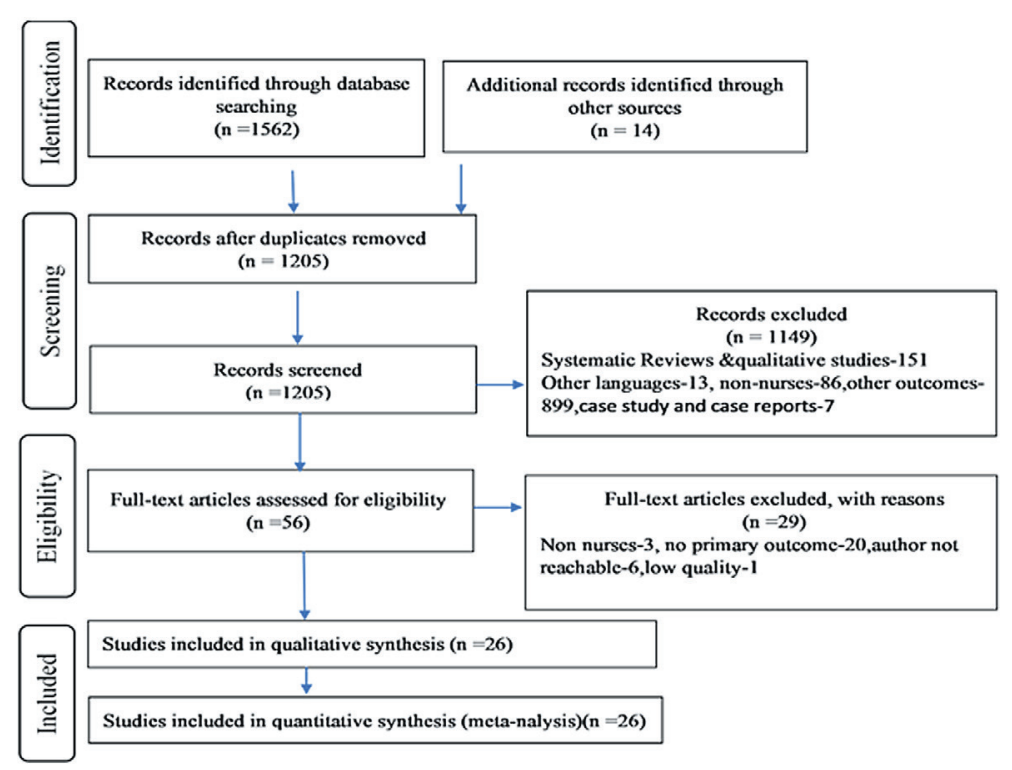

Figure 1. PRISMA flowchart depicting the selection process of included studies.

The essential attributes in the studies are displayed in Table 1. From March to August 2020, these studies were published with prevalence reports from different regions of the world namely; Asia (14), Europe (7), the Eastern Mediterranean Region (4) and the Americas (1). All the studies were cross-sectional, conducted among nurses in hospitals, via online web-based surveys while a single study was conducted nationally [33]. The majority of the reports focused on nurses as the primary sample (53.8\%), while other health care professionals along with nurses accounted for the remainder $(46.2 \%)$. The total sample size was 17100 ranging from 45 [41] to 4692 [12]. Furthermore, for each of the mental ailments; anxiety, stress, depression, PTSD, insomnia, the percentage of nurses from the overall study sample $(17,100)$ was $79.8 \%, 24.6 \%, 72.0 \%, 3.7 \%, 1.5 \%$, respectively. Various validated scales with specific cut-off were used in our study such as; Generalised Anxiety Disorder, Zung's Self-Rating Anxiety Scale, COVD-19 Anxiety Scale (CAS), The State-Trait Anxiety Inventory, Hospital Anxiety and Depression Scale, Stanford Acute Stress Reaction Questionnaire, Global stress Index (GSI), Self-reported Stressor and Incidence Questionnaire, Perceived Stress Scale, Hamilton Depression Rating Scale, Zung's Self-Rating Depression Scale, Patient Health Questionnaire (PHQ-9), Depression Anxiety Stress Scale (DASS-21), Impact of Event Scale, PTSD Checklist-Civilian version and Pittsburgh Sleep Quality Index. However, no report has used formal psychiatric interviews to evaluate the presence of an actual illness or its severity. The participants' age ranged from $21-45$ years and most were females (91.8\%), while $8.2 \%$ were males. The majority of the sample was married (63.1\%) and 36.9\% were single.

\section{Quality evaluation}

The methodological quality of the studies was assessed by two reviewers using Loney criteria [21]. Any discrepancy in the scoring between the two reviewers was resolved by mutual discussion and accord. The quality score ranged from 5-8 with 6 as the median score (interquartile range: 5-7) after the exclusion of a single study with a low-quality score of 4 . Eventually, twenty-six studies with moderate or high quality were included for final analysis (Table S2 in the Online Supplementary Document).

\section{Primary mental health outcomes}

Anxiety: A total of 21 studies [9,10,12,28-34,37,38,40-48] assessed the prevalence of anxiety among nurses and its severity level was appraised by eight studies $[10,31,33,34,37,40,46,48]$. The overall pooled prevalence of anxiety was 33\% (95\% CI=24\%-43\%) with substantial heterogeneity $\left(I^{2}=99.4 \%, P<0.01\right)$ (Figure $2)$. Further, mild anxiety was more $(24.8 \%)$ common compared to moderate $(12.9 \%)$, severe $(7.1 \%)$ and extreme severe anxiety (2\%) estimated at a significant heterogeneity level. Moreover, no significant differences in the prevalence of anxiety were found in the subgroup analysis; males (27\%), females (33.3\%) and married (35.7\%), single (31.5\%) (Table 2). The regional analysis has shown that the prevalence of anxiety was more 
Table 1. Characteristics of included studies

\begin{tabular}{|c|c|c|c|c|c|c|c|c|c|c|}
\hline $\begin{array}{l}\text { PRIMARY AUTHOR, MONTH, } \\
\text { YEAR \& COUNTRY }\end{array}$ & DESIGN\& SETTING & $\begin{array}{l}\text { SAMPLE } \\
\text { SIZE } \\
\text { (NURSES) }\end{array}$ & EVALUATING SCORE & $\begin{array}{l}\text { PREVALENCE } \\
\text { OF } \\
\text { ANXIETY }\end{array}$ & $\begin{array}{l}\text { Prevalence } \\
\text { OF } \\
\text { StRESS }\end{array}$ & $\begin{array}{l}\text { Prevalence } \\
\text { OF } \\
\text { DEPRESSION }\end{array}$ & PTSD & INSOMNIA & $\begin{array}{l}\text { SIGNIFICANT RISK FACTORS OF MENTAL } \\
\text { AILMENTS* }\end{array}$ & $\begin{array}{l}\text { QUALITY } \\
\text { SCORE' }\end{array}$ \\
\hline $\begin{array}{l}\text { Dal Bosco et al., May 2020, } \\
\text { Brazil [9] }\end{array}$ & $\begin{array}{l}\text { Cross-Sectional, } \\
\text { Regional University } \\
\text { Hospital }\end{array}$ & 88 & HAD-A\&HAD-D>8 & $\begin{array}{c}49 \% \\
(43 / 88)\end{array}$ & NA & $\begin{array}{c}25 \% \\
(22 / 88)\end{array}$ & NA & NA & $\begin{array}{l}\text { Anxiety: } 31-40 \text { y, married women \&working } \\
\text { in critical sectors; Depression: } 21-30 \text { y, single } \\
\text { and women working in critical sectors-ICU, } \\
\text { surgery and emergency }\end{array}$ & 6 \\
\hline $\begin{array}{l}\text { Darija Salopek-Ziha et al., } \\
\text { June 2020, Croatia [10] }\end{array}$ & $\begin{array}{l}\text { Cross-sectional, } \\
\text { general hospital }\end{array}$ & 71 & $\begin{array}{l}\text { DASS- } 21, \\
\text { Anxiety } \geq 10, \\
\text { Stress } \geq 15 \\
\text { Depression } \geq 10\end{array}$ & $\begin{array}{c}24 \% \\
(17 / 71)\end{array}$ & $\begin{array}{l}21.1 \% \\
(15 / 71)\end{array}$ & $\begin{array}{l}26.8 \% \\
(19 / 71)\end{array}$ & NA & NA & NA & 5 \\
\hline $\begin{array}{l}\text { Eva-Maria Skoda et al., } \\
\text { March 2020, Germany [28] }\end{array}$ & $\begin{array}{l}\text { Cross-sectional study, } \\
\text { nationwide }\end{array}$ & 1511 & GAD- $7 \geq 10$ & $\begin{array}{c}11.4 \% \\
(172 / 1511)\end{array}$ & NA & NA & NA & NA & NA & 6 \\
\hline $\begin{array}{l}\text { Zerbini et al.,2020, Germany } \\
\text { [29] }\end{array}$ & $\begin{array}{l}\text { Cross-sectional } \\
\text { study,1 hospital }\end{array}$ & 75 & $\begin{array}{l}\text { GAD- } 7 \geq 10, \text { PHQ- } \\
\text { stress } \geq 5, \text { PHQ- } 9 \geq 10\end{array}$ & $\begin{array}{c}16 \% \\
(12 / 75)\end{array}$ & $\begin{array}{l}45.3 \% \\
(34 / 75)\end{array}$ & $\begin{array}{l}29.3 \% \\
(22 / 75)\end{array}$ & NA & NA & $\begin{array}{l}\text { Anxiety, stress, depression: caring for } \\
\text { COVID-19 patients and working in COVID-19 } \\
\text { wards. }\end{array}$ & 5 \\
\hline $\begin{array}{l}\text { Szepietowski et al., June } \\
\text { 2020, Poland [30] }\end{array}$ & $\begin{array}{l}\text { Cross-Sectional study, } \\
\text { hospital }\end{array}$ & 62 & $\begin{array}{l}\text { GAD-7 } \geq 5 \text {, PHQ- } \\
9 \geq 10\end{array}$ & $\begin{array}{l}\text { GAD- } \\
7: 46.7 \% \\
(29 / 62) \\
\end{array}$ & NA & $\begin{array}{l}\text { PHQ-9:21\% } \\
\quad(13 / 62)\end{array}$ & NA & NA & NA & 5 \\
\hline $\begin{array}{l}\text { Bachilo et al., July 2020, } \\
\text { Russia [31] }\end{array}$ & $\begin{array}{l}\text { Cross-Sectional study, } \\
\text { Hospital }\end{array}$ & 139 & $\mathrm{GAD} \geq 5, \mathrm{PHQ} \geq 5$ & $\begin{array}{l}39.6 \% \\
(55 / 139) \\
\end{array}$ & NA & $\begin{array}{c}50.4 \% \\
(70 / 139)\end{array}$ & NA & NA & NA & 7 \\
\hline $\begin{array}{l}\text { Giusti et al., July 2020, Italy } \\
\text { [32] }\end{array}$ & $\begin{array}{l}\text { Cross-Sectional Study, } \\
\text { Hospital }\end{array}$ & 86 & $\begin{array}{l}\text { DASS } 21 \text {, } \\
\text { Depression } \geq 4, \\
\text { Stress } \geq 7, \text { IES }>9 \\
\text { Anxiety } \geq 3\end{array}$ & $\begin{array}{l}41.9 \% \\
(36 / 86)\end{array}$ & $\begin{array}{l}37.2 \% \\
(32 / 86)\end{array}$ & $\begin{array}{l}26.7 \% \\
(23 / 86)\end{array}$ & $\begin{array}{l}39.5 \% \\
(34 / 86)\end{array}$ & NA & Working in COVID-19 ward. & 5 \\
\hline $\begin{array}{l}\text { Shahrour et al., August } \\
\text { 2020, Jordan [11] }\end{array}$ & $\begin{array}{l}\text { Cross-sectional } \\
\text { survey, hospitals. }\end{array}$ & 448 & $\begin{array}{l}\text { SASRQ } \geq 56, \text { ASD, } \\
\text { GSI }>50 \text {-psycholoical } \\
\text { distress }\end{array}$ & NA & $\begin{array}{l}\text { ASD-64.1\% } \\
(287 / 448) \\
\text { GSI-41.1\% } \\
(184 / 448) \\
\end{array}$ & NA & NA & NA & $\begin{array}{l}\text { Psychological distress, younger nurses, lower } \\
\text { coping self-efficacy, higher ASD. }\end{array}$ & 5 \\
\hline $\begin{array}{l}\text { Naser et al., June 2020, } \\
\text { Jordan [33] }\end{array}$ & $\begin{array}{l}\text { Cross-sectional study, } \\
\text { nationwide }\end{array}$ & 151 & GAD-7 $\geq 5$, PHQ- $9 \geq 5$ & $\begin{array}{c}79.5 \% \\
(120 / 151)\end{array}$ & NA & $\begin{array}{c}80.8 \% \\
(125 / 151)\end{array}$ & NA & NA & NA & 5 \\
\hline $\begin{array}{l}\text { Deying et al., June 2020, } \\
\text { China [34] }\end{array}$ & $\begin{array}{l}\text { Cross-sectional study, } \\
\text { two hospitals. }\end{array}$ & 2014 & $S R A \geq 50, S D S \geq 23$ & $\begin{array}{c}41.4 \% \\
(833 / 2014) \\
\end{array}$ & NA & $\begin{array}{c}43.6 \% \\
(878 / 2014) \\
\end{array}$ & NA & NA & $\begin{array}{l}\text { Anxiety and depression: low self-efficacy, } \\
\text { resilience and social support. }\end{array}$ & 8 \\
\hline $\begin{array}{l}\text { Su Hong et al., July 2020, } \\
\text { China [12] }\end{array}$ & $\begin{array}{l}\text { Cross-sectional } \\
\text { survey; } 42 \\
\text { Government } \\
\text { Hospitals. }\end{array}$ & 4692 & $\begin{array}{l}\text { GAD- } 7 \geq 10, \text { PHQ- } \\
9 \geq 10\end{array}$ & $\begin{array}{c}8.1 \% \\
(379 / 4692)\end{array}$ & NA & $\begin{array}{c}9.4 \% \\
(442 / 4692)\end{array}$ & NA & NA & $\begin{array}{l}\text { Depression: Single, no support from family } \\
\text { and hospital authority, being discriminated; } \\
\text { Job-related stressors: High workload, being } \\
\text { quarantined and } \\
\text { impaired work ability. }\end{array}$ & 7 \\
\hline $\begin{array}{l}\text { Chen et al., July 2020, } \\
\text { China [35] }\end{array}$ & $\begin{array}{l}\text { Cross-sectional } \\
\text { prospective survey, } \\
\text { hospital. }\end{array}$ & 92 & SRISQ & NA & $\begin{array}{c}7-10 \\
\text { d- }(68.5 \%) \\
63 / 92\end{array}$ & NA & NA & NA & Working in isolation wards. & 5 \\
\hline $\begin{array}{l}\text { Yifang Zhou et al., June } \\
2020 \text { China [36] }\end{array}$ & $\begin{array}{l}\text { Cross-sectional } \\
\text { survey, community. }\end{array}$ & 1614 & $\mathrm{PSQI} \geq 7$ & NA & NA & NA & NA & $\begin{array}{c}19.5 \% \\
(314 / 1614)\end{array}$ & $\begin{array}{l}\text { Old age, Working in the emergency medical } \\
\text { team. }\end{array}$ & 5 \\
\hline $\begin{array}{l}\text { Zhi-hao et al., May 2020, } \\
\text { China [37] }\end{array}$ & $\begin{array}{l}\text { Cross-sectional } \\
\text { survey, hospital. }\end{array}$ & 100 & $\begin{array}{l}\text { GAD-7 } \geq 4, \text { PSQI } \geq 7, \\
\text { PHQ- } 9 \geq 4\end{array}$ & $\begin{array}{c}40 \% \\
(40 / 100) \\
\end{array}$ & NA & $\begin{array}{c}46 \% \\
(46 / 100) \\
\end{array}$ & NA & $\begin{array}{c}60 \% \\
(60 / 100) \\
\end{array}$ & $\begin{array}{l}\text { Depression: High anxiety. poor sleep quality } \\
\text { and only child in the family. }\end{array}$ & 5 \\
\hline
\end{tabular}




\begin{tabular}{|c|c|c|c|c|c|c|c|c|c|c|}
\hline $\begin{array}{l}\text { PRIMARY AUTHOR, MONTH, } \\
\text { YEAR \& COUNTRY }\end{array}$ & DESIGN\& SETTING & $\begin{array}{l}\text { SAMPLE } \\
\text { SIZE } \\
\text { (NURSES) }\end{array}$ & EVALUATING SCORE & $\begin{array}{l}\text { PreVAlence } \\
\text { OF } \\
\text { ANXIETy }\end{array}$ & $\begin{array}{l}\text { Prevalence } \\
\text { OF } \\
\text { StRESS }\end{array}$ & $\begin{array}{l}\text { Prevalence } \\
\text { OF } \\
\text { DEPRESSION }\end{array}$ & PTSD & INSOMNIA & $\begin{array}{l}\text { SIGNIFICANT RISK FACTORS OF MENTAL } \\
\text { AILMENTS* }\end{array}$ & $\begin{array}{l}\text { QUALITY } \\
\text { SCORE' }\end{array}$ \\
\hline $\begin{array}{l}\text { Yu-Xin Zhan et al.,2020, } \\
\text { China [38] }\end{array}$ & $\begin{array}{l}\text { Cross-sectional, } \\
\text { hospital. }\end{array}$ & 2667 & $\begin{array}{l}\text { GAD- } 7 \geq 5 \text {, CPSS } \geq 25 \\
\text { PHQ- } 9 \geq 5\end{array}$ & $\begin{array}{c}39.8 \% \\
(1062 / 2667) \\
\end{array}$ & $\begin{array}{c}48.7 \% \\
(1298 / 2667) \\
\end{array}$ & $\begin{array}{c}54.7 \% \\
(1458 / 2667) \\
\end{array}$ & NA & NA & NA & 7 \\
\hline $\begin{array}{l}\text { Ying An et al., July 2020, } \\
\text { China [39] }\end{array}$ & $\begin{array}{l}\text { Cross-sectional } \\
\text { survey, emergency } \\
\text { department. }\end{array}$ & 1103 & PHQ-9 $\geq 5$ & NA & NA & $\begin{array}{c}43.7 \% \\
(481 / 1103)\end{array}$ & NA & NA & $\begin{array}{l}\text { Depression: COVID Centre, current smokers, } \\
\text { Emergency department nurses. }\end{array}$ & 8 \\
\hline $\begin{array}{l}\text { Ya-Xi Wang et al., May } \\
\text { 2020, China [13] }\end{array}$ & $\begin{array}{l}\text { Cross-sectional study, } \\
\text { three hospitals. }\end{array}$ & 202 & PCL-C $\geq 38$ & NA & NA & NA & $\begin{array}{c}16.8 \% \\
(34 / 202)\end{array}$ & NA & $\begin{array}{l}\text { PTSD: Female, negative coping, low job } \\
\text { satisfaction. }\end{array}$ & 6 \\
\hline $\begin{array}{l}\text { Ruilin Li et al., June 2020, } \\
\text { China [40] }\end{array}$ & $\begin{array}{l}\text { Cross-sectional study, } \\
\text { COVID hospital. }\end{array}$ & 176 & $\mathrm{HAMA} \geq 7$ & $\begin{array}{c}77.3 \% \\
(136 / 176)\end{array}$ & NA & NA & NA & NA & $\begin{array}{l}\text { Anxiety: long working hours, wearing } \\
\text { protective equipment, female and great } \\
\text { workload., working in COVID-19 designated } \\
\text { hospitals. }\end{array}$ & 6 \\
\hline $\begin{array}{l}\text { Cuong Do Duy et al., July } \\
\text { 2020, Vietnam [41] }\end{array}$ & $\begin{array}{l}\text { Cross-sectional } \\
\text { survey, COVID } \\
\text { hospital. }\end{array}$ & 45 & $\begin{array}{l}\text { DAS-21, Anxiety } \geq 3, \\
\text { Stress } \geq 4 \\
\text { Depression } \geq 4\end{array}$ & $\begin{array}{l}11.1 \% \\
(5 / 45)\end{array}$ & $2.2 \%(1 / 45)$ & $\begin{array}{l}11.1 \% \\
(5 / 45)\end{array}$ & NA & NA & NA & 5 \\
\hline $\begin{array}{l}\text { Saricam et al., July 2020, } \\
\text { Turkey [42] }\end{array}$ & $\begin{array}{l}\text { Cross-sectional study, } \\
\text { hospital. }\end{array}$ & 123 & $\mathrm{STAI} \geq 57$ & $\begin{array}{c}46.3 \% \\
(57 / 123)\end{array}$ & NA & NA & NA & NA & $\begin{array}{l}\text { Anxiety: Advancing age and years of } \\
\text { experience, working in wards and having } \\
\text { a child; Working in pandemic and normal } \\
\text { wards. }\end{array}$ & 8 \\
\hline $\begin{array}{l}\text { Lee et al., 2020, Singapore } \\
\text { [43] }\end{array}$ & $\begin{array}{l}\text { Cross-sectional study, } \\
\text { tertiary hospital. }\end{array}$ & 155 & $\begin{array}{l}\text { HADS-A }>10 \\
\text { HADS-D }>10\end{array}$ & $\begin{array}{l}33.5 \% \\
(52 / 155)\end{array}$ & NA & $\begin{array}{c}31.6 \% \\
(49 / 155)\end{array}$ & NA & NA & $\begin{array}{l}\text { Psychological distress: Multiple co-morbidities } \\
\text { in staff, COVID care, quarantine order, } \\
\text { redeployment outside normal professional } \\
\text { boundaries. }\end{array}$ & 8 \\
\hline $\begin{array}{l}\text { Labrague et al.,2020, } \\
\text { Philippines [44] }\end{array}$ & $\begin{array}{l}\text { Cross-sectional study, } \\
\text { hospitals. }\end{array}$ & 325 & $\mathrm{CAS} \geq 9$ & $\begin{array}{c}37.8 \% \\
(123 / 325) \\
\end{array}$ & NA & NA & NA & NA & $\begin{array}{l}\text { Anxiety: Less social, organizational support } \\
\text { and personal resilience. }\end{array}$ & 8 \\
\hline $\begin{array}{l}\text { Abdallah Badahdah et } \\
\text { al.,2020, Oman [45] }\end{array}$ & $\begin{array}{l}\text { Cross-sectional } \\
\text { study, } 10 \text { hospitals. }\end{array}$ & 315 & GAD- $7 \geq 10$, PSS $\geq 24$ & $\begin{array}{c}11.4 \% \\
(36 / 315) \\
\end{array}$ & $\begin{array}{c}58.7 \% \\
(185 / 315) \\
\end{array}$ & NA & NA & NA & Stress: Females, COVID Centre & 5 \\
\hline $\begin{array}{l}\text { Pouralizadeh et al., August } \\
\text { 2020, Iran [46] }\end{array}$ & $\begin{array}{l}\text { Cross-sectional study, } \\
25 \text { hospitals. }\end{array}$ & 441 & $\begin{array}{l}\text { GAD-7 } \geq 10, \text { PHQ- } \\
9 \geq 10\end{array}$ & $\begin{array}{c}38.7 \% \\
(171 / 441)\end{array}$ & NA & $\begin{array}{c}37.4 \% \\
(165 / 441)\end{array}$ & NA & NA & $\begin{array}{l}\text { Anxiety: Female nurses, COVID hospital, } \\
\text { Suspected infection, lack of access to PPE; } \\
\text { Depression-Chronic illness, suspected or } \\
\text { positive cases, no access to personal protective } \\
\text { equipment and female nurses }\end{array}$ & 8 \\
\hline $\begin{array}{l}\text { Wilson et al., July 2020, } \\
\text { India [47] }\end{array}$ & $\begin{array}{l}\text { Cross-sectional study, } \\
\text { COVID-19 hospital. }\end{array}$ & 55 & $\begin{array}{l}\text { GAD- } 7 \geq 10, \text { PSS } \geq 14, \\
\text { PHQ } 9 \geq 10\end{array}$ & $\begin{array}{l}21.8 \% \\
(12 / 55) \\
\end{array}$ & $\begin{array}{c}80 \% \\
(44 / 55) \\
\end{array}$ & $\begin{array}{l}14.5 \% \\
(8 / 55) \\
\end{array}$ & NA & NA & Anxiety, Stress, Depression: female gender. & 7 \\
\hline $\begin{array}{l}\text { Chew et al., 2020, India and } \\
\text { Singapore [48] }\end{array}$ & $\begin{array}{l}\text { Cross-sectional study, } \\
\text { tertiary hospitals. }\end{array}$ & 350 & $\begin{array}{l}\text { DASS-21, IES, } \\
\text { Anxiety }>7, \text { Stress }>14, \\
\text { Depression }>9, \\
\text { PTSD }>24\end{array}$ & $\begin{array}{c}9.4 \% \\
(33 / 350)\end{array}$ & $\begin{array}{c}3.7 \% \\
(13 / 350)\end{array}$ & $\begin{array}{c}7.1 \% \\
(25 / 350)\end{array}$ & $\begin{array}{c}6 \% \\
(21 / 350)\end{array}$ & NA & $\begin{array}{l}\text { Anxiety, Stress, Depression, PTSD: Physical } \\
\text { Symptoms (sore throat, breathlessness, cough, } \\
\text { lethargy, myalgia, fever). }\end{array}$ & \\
\hline
\end{tabular}

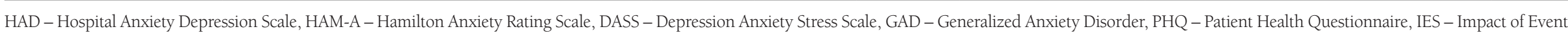

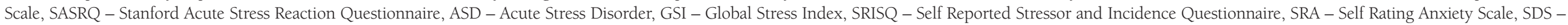
Self Rating Depression Scale, PSQI - Pittsburg Sleep Quality Index, PSS - Perceived Stress Scale, CAS - COVID-19 Anxiety Scale, PPE - personal protective equipment

$*<<0.05$ or odds ratio $>1$

$\uparrow$ The Loney criteria [21]. 
Studies

DalBosco EB, etal.,May, Brazil 2020

Darija Salopek-iha,etal.,June,Croatia 2020

Eva-Maria Skoda,etal.,March,Germany 2020

Zerbini G,etal.,Germany 2020

Jacek C Szepietowski, etal.,June,Poland 2020

Bachilo EV,et al.,July, Russia 2020

Giusti EM,etal.,July,Italy 2020

Naser AY, etal., June, Jordan 2020

Deying Hu, etal., June.China 2020

Su Hong, etal,July,China 2020

Zhi-hao Tu,etal.,May,China 2020

Yu-Xin Zhan,etal.,China 2020

Ruilin Li,etal.,June,China 2020

Cuong Do Duy,etal.,July, Vietnam 2020

Saricam M,etal., July, Turkey 2020

Lee MC, etal.,Singapore 2020

Leodoro J Labrague,etal.,Philippines 2020

Abdallah Badahdah,etal.,Oman 2020

Pouralizadeh M,etal.,August, Iran 2020

Wilson W.etal.,July, India 2020

Chew NWS,etal.,India and Singapore 2020

Overall ( $\left({ }^{\wedge} 2=99.17 \%, P<0.01\right)$
Estimate (958 C.I.) Ev/N

$0.49(0.38,0.59) \quad 43 / 88$

$0.24(0.15,0.35) \quad 17 / 71$

$0.11(0.10,0.13) \quad 172 / 1511$

$0.16(0.08,0.25) \quad 12 / 75$

$0.47(0.34,0.59) \quad 29 / 62$

$0.40(0.32,0.48) \quad 55 / 139$

$0.42(0.32,0.52) \quad 36 / 86$

$0.79(0.73,0.86) \quad 120 / 151$

$0.41(0.39,0.44) \quad 833 / 2014$

$0.08(0.07,0.09) \quad 379 / 4692$

$0.40(0.31,0.50) \quad 40 / 100$

$0.40(0.38,0.42) 1062 / 2667$

$0.77(0.71,0.83) \quad 136 / 176$

$0.11(0.03,0.22) \quad 5 / 45$

$0.46(0.38,0.55) \quad 57 / 123$

$0.34(0.26,0.41) \quad 52 / 155$

$0.38(0.33,0.43) \quad 123 / 325$

$0.11(0.08,0.15) \quad 36 / 315$

$0.39(0.34,0.43) \quad 171 / 441$

$0.22(0.12,0.34) \quad 12 / 55$

$0.09(0.07,0.13) \quad 33 / 350$

$0.33(0.24,0.43) 3423 / 13641$

Figure 2. Pooled prevalence of anxiety among nurses $(\mathrm{Q}=2467.18, \mathrm{df}=20)$.

Table 2. Pooled prevalence of various subgroup categories of mental health outcomes among nurses

\begin{tabular}{|c|c|c|c|c|c|c|c|c|c|c|}
\hline \multirow[b]{2}{*}{$\begin{array}{l}\text { MENTAL } \\
\text { HEALTH } \\
\text { OUTCOME }\end{array}$} & \multirow[b]{2}{*}{ VARIABLE } & \multirow[b]{2}{*}{$\begin{array}{l}\text { No. OF } \\
\text { STUDIES }\end{array}$} & \multirow[b]{2}{*}{$\begin{array}{l}\text { No. OF } \\
\text { PARTICl- } \\
\text { PANTS }\end{array}$} & \multirow[b]{2}{*}{$\begin{array}{l}\text { No. OF } \\
\text { POSITIVE } \\
\text { CASES }\end{array}$} & \multirow[b]{2}{*}{$X-2(P)$} & \multicolumn{5}{|c|}{ HETEROGENEITY } \\
\hline & & & & & & $\begin{array}{l}\text { Estimate, \% } \\
\text { (confidence } \\
\text { interval) }\end{array}$ & $\mathrm{I}^{2}$ & $P$ & $\mathrm{Tau}^{2}$ & $\mathcal{Q}(\mathrm{df})$ \\
\hline \multirow{8}{*}{ Anxiety } & Mild anxiety & 6 & 3195 & 832 & \multirow{4}{*}{$(0.00001)$} & $24.8(13.7-37.9)$ & 97.75 & $<0.001$ & 0.03 & $222.06(5)$ \\
\hline & Moderate anxiety & 7 & 3266 & 399 & & $12.9(8.2-18.4)$ & 91.85 & $<0.001$ & 0.01 & $73.856(6)$ \\
\hline & Severe anxiety & 8 & 3442 & 230 & & $7.1(2.5-13.7)$ & 96.53 & $<0.001$ & 0.02 & $201.71(7)$ \\
\hline & Ex- severe anxiety & 2 & 421 & 5 & & $2(0-7)$ & 77.79 & $<0.001$ & 0.01 & $4.50(1)$ \\
\hline & Males & 4 & 206 & 34 & \multirow{2}{*}{$\begin{array}{c}3.8 \\
(<0.06)\end{array}$} & $27(4-58)$ & 92.22 & $<0.001$ & 0.09 & $38.57(3)$ \\
\hline & Females & 4 & 5138 & 616 & & $33.3(10.4-61.6)$ & 99.17 & $<0.001$ & 0.08 & $359.42(3)$ \\
\hline & Married & 4 & 3456 & 428 & \multirow{2}{*}{$\begin{array}{c}0.93 \\
(0.34)\end{array}$} & $35.7(11.8-64.1)$ & 98.85 & $<0.02$ & 0.08 & $262.93(3)$ \\
\hline & Single & 4 & 1872 & 215 & & $31.5(8.5-60.7)$ & 97.63 & $<0.02$ & 0.09 & $126.57(3)$ \\
\hline \multirow{4}{*}{ Stress } & Mild stress & 2 & 421 & 10 & \multirow{4}{*}{$\begin{array}{c}6.4 \\
(0.1)\end{array}$} & $2.9(0.2-8)$ & 68.66 & $<0.074$ & 0.01 & $3.19(1)$ \\
\hline & Moderate stress & 2 & 421 & 10 & & $2.9(0.2-8)$ & 68.66 & 0.074 & 0.01 & $3.19(1)$ \\
\hline & Severe stress & 2 & 421 & 6 & & $2.3(0-13.2)$ & 90.92 & $<0.001$ & 0.02 & $11.01(1)$ \\
\hline & Extreme severe stress & 2 & 421 & 2 & & $0.8(0-5.3)$ & 76.91 & 0.037 & 0.01 & $4.33(1)$ \\
\hline \multirow{8}{*}{ Depression } & Mild depression & 7 & 4269 & 1228 & \multirow{4}{*}{$(<0.0000)$} & $23.8(15.2-33.6)$ & 97.56 & $<0.001$ & 0.02 & $246.514(6)$ \\
\hline & Moderate depression & 7 & 4269 & 445 & & $11(7.1-15.6)$ & 93.24 & $<0.001$ & 0.01 & $88.86(6)$ \\
\hline & Severe depression & 7 & 4269 & 141 & & $4(1.6-7.3)$ & 93.95 & $<0.001$ & 0.01 & $99.167(6)$ \\
\hline & Extremely severe depression & 4 & 1663 & 31 & & $1.7(0.4-3.6)$ & $<0.001$ & 0.002 & & $10.614(3)$ \\
\hline & Males & 4 & 276 & 69 & \multirow{2}{*}{$\begin{array}{c}11 \\
(0.0009)\end{array}$} & $22.5(2.8-51.6)$ & 94.54 & $<0.001$ & 0.08 & $54.98(3)$ \\
\hline & Females & 4 & 6048 & 1042 & & $27.9(8.5-53)$ & 99.55 & $<0.001$ & 0.07 & $666.65(3)$ \\
\hline & Married & 4 & 4090 & 692 & \multirow{2}{*}{$\begin{array}{c}2.7 \\
(0.09)\end{array}$} & $26.9(7-53.5)$ & 99.42 & $<0.001$ & 0.08 & $522.45(3)$ \\
\hline & Single & 4 & 2218 & 412 & & $28.4(9.6-52.1)$ & 98.56 & $<0.001$ & 0.06 & $209.02(3)$ \\
\hline PTSD & & 3 & 638 & 89 & & $18.6(4.8-38.3)$ & 96.43 & $<0.001$ & 0.04 & $56.02(2)$ \\
\hline Insomnia & & 2 & 1714 & 374 & & $38.3(5.8-78.6)$ & 98.56 & $<0.001$ & 0.09 & $98.558(1)$ \\
\hline
\end{tabular}

PTSD - posttraumatic stress disorder

*P $P<0.05$.

among nurses from the Eastern Mediterranean region (41.9\%) compared to the European region (30.6\%) and the combined prevalence reported from Western Pacific and South-East Asian regions (30.9\%) (Table 3). The prevalence of mental health outcomes reported form Western Pacific and South-East Asian Regions were combined since our study included an individual study which was conducted across two different countries [48].

Stress: The overall prevalence of stress among nurses in the ten included studies $[10,11,29,32,35,38,41,45,47,48]$ was $40.6 \%(25.4 \%-56.8 \%)$ with significant heterogeneity $\left(I^{2}=98.6 \%, P<0.001\right)$ (Figure 3$)$. A comparative proportion of prevalence was found among different severity levels; mild (2.9\%), moderate (2.9\%), severe (2.3\%) 
Table 3. Pooled estimate of mental health outcomes among nurses in different regions of the world

\begin{tabular}{|c|c|c|c|c|c|c|c|c|c|}
\hline \multirow{2}{*}{ REGION } & \multirow{2}{*}{$\begin{array}{l}\text { MENTAL } \\
\text { HEALTH } \\
\text { OUTCOMES }\end{array}$} & \multirow{2}{*}{$\begin{array}{l}\text { No. OF } \\
\text { STUDIES }\end{array}$} & \multirow{2}{*}{$\begin{array}{l}\text { No. OF } \\
\text { PARTICI- } \\
\text { PANTS }\end{array}$} & \multirow{2}{*}{$\begin{array}{l}\text { No. OF } \\
\text { POSITIVES }\end{array}$} & \multirow{2}{*}{$\begin{array}{l}\text { ESTIMATE, \% } \\
\text { (CONFIDENCE } \\
\text { INTERVAL) }\end{array}$} & \multicolumn{4}{|c|}{ HETEROGENEITY } \\
\hline & & & & & & $\mathrm{I}^{2}$ & $P$-value & $\mathrm{Tau}^{2}$ & $\mathcal{Q}(\mathrm{df})$ \\
\hline \multirow{3}{*}{$\begin{array}{l}\text { European } \\
\text { region }\end{array}$} & Anxiety & 7 & 2067 & 374 & $30.5(16.7-46.3)$ & 96.58 & $<0.001$ & 0.05 & $175.68(6)$ \\
\hline & Stress & 3 & 232 & 81 & $34.2(21.2-48.6)$ & 80.19 & $<0.001$ & 0.01 & $10.01(2)$ \\
\hline & Depression & 5 & 433 & 147 & $30.9(20.4-42.5)$ & 83.82 & $<0.001$ & 0.02 & $24.73(4)$ \\
\hline \multirow{5}{*}{$\begin{array}{l}\text { Western } \\
\text { Pacific \& } \\
\text { South-East } \\
\text { region }\end{array}$} & Anxiety & 10 & 10579 & 2720 & $30.9(17.2-46.5)$ & 99.53 & $<0.001$ & 0.07 & $1931.52(9)$ \\
\hline & Stress & 4 & 3165 & 1418 & $47.2(14.7-81)$ & 99.36 & $<0.001$ & 0.13 & $467.66(3)$ \\
\hline & Depression & 9 & 11181 & 3392 & $27.4(13-44.7)$ & 99.67 & $<0.001$ & 0.07 & $2411.47(8)$ \\
\hline & PTSD & 2 & 552 & 55 & $10.7(2.5-23.5)$ & 93.63 & $<0.001$ & 0.01 & $15.70(1)$ \\
\hline & Insomnia & 2 & 1714 & 374 & $38.3(5.8-78.6)$ & 98.56 & $<0.001$ & 0.09 & $69.34(1)$ \\
\hline \multirow{3}{*}{$\begin{array}{l}\text { Eastern } \\
\text { Mediterranean } \\
\text { region }\end{array}$} & Anxiety & 3 & 907 & 327 & $41.9(10.7-77.3)$ & 99.16 & $<0.001$ & 0.11 & $273.25(2)$ \\
\hline & Stress & 2 & 763 & 472 & $61.6(56.4-66.8)$ & 54.97 & 0.136 & 0.001 & $2.22(1)$ \\
\hline & Depression & 2 & 592 & 290 & $61.2(16.9-96.2)$ & 99.04 & $<0.001$ & 0.12 & $104.64(1)$ \\
\hline
\end{tabular}

Studies

Darija Salopek-Ziha, etal., June,Croatia 2020

Zerbini G,etal.,Germany 2020

Giusti EM,etal.,July,Italy 2020

Shahrour G,et al.,August, Jordan 2020

Chen H,etal.,July, China 2020

Yu-Xin Zhan,etal.,China 2020

Cuong Do Duy,etal.,July,Vietnam 2020

Abdallah Badahdah,etal.,Oman 2020

Wilson W,etal.,July,India 2020

Chew NWS, etal.,India and Singapore 2020

Overall (I^2=98.59\%, P< 0.001$)$
Estimate (958 C.I.) Ev/N

$0.211(0.123,0.315) \quad 15 / 71$

$0.453(0.342,0.567) \quad 34 / 75$

$0.372(0.273,0.477) \quad 32 / 86$

$0.641(0.596,0.684) \quad 287 / 448$

$0.685(0.586,0.776) \quad 63 / 92$

$0.487(0.468,0.506) 1298 / 2667$

$0.022(0.000,0.093) \quad 1 / 45$

$0.587(0.532,0.641) \quad 185 / 315$

$0.800(0.683,0.897) \quad 44 / 55$

$0.037(0.020,0.060) \quad 13 / 350$

$0.406(0.254,0.568) 1972 / 4204$

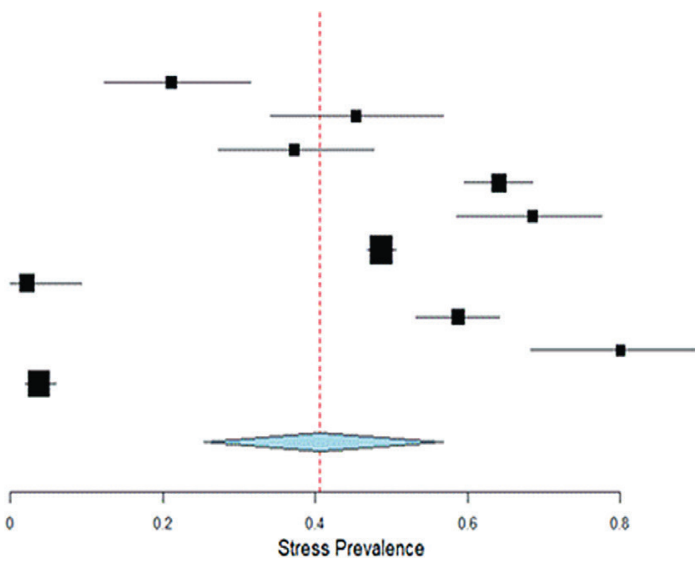

Figure 3. Pooled prevalence of stress among nurses $(\mathrm{Q}=636.58, \mathrm{df}=9)$.

and extreme severe (0.8\%) (Table 2). Besides, the Eastern Mediterranean region showed a higher prevalence of stress (61.6\%) compared to Europe (34.2\%) and the combined prevalence reported from Western Pacific and South-East Asian regions (47.2\%) (Table 3).

Depression: Pooled prevalence of depression from 17 studies [9,10,12,29-34,37-39,41,43,46-48] was 32\% (95\% CI $=21 \%-44 \%)$ with significant heterogeneity $\left(I^{2}=99.4 \%, P<0.01\right)$ (Figure 4). Different estimates of

Studies

DalBosco EB, etal.,May, Brazil 2020 Darja Salopek-Ziha,etal.,June,Croatia 2020 Zerbini G,etal.,Germany 2020

Jacek C Szepietowski, etal., June,Poland 2020

Bachilo EV,et al.,July,Russia 2020

Giusti EM,etal., July, Italy 2020

Naser AY, etal.,June, Jordan 2020

Deying Hu,etal. June.China 2020

Su Hong, etal, July, China 2020

Zhi-hao Tu,etal.,May, China 2020

Yu-Xin Zhan,etal.,China 2020

Ying An, etal.,July, China 2020

Cuong Do Duy,etal., July, Vietnam 2020

Lee MC, etal.,Singapore 2020

Pouralizadeh M,etal.,August, Iran 2020

Wilson W,etal.,July,India 2020

Chew NWS, etal.,India and Singapore 2020

Overall $\left(\left.\right|^{\wedge} 2=99.4 \%, P<0.01\right)$
Estimate (958 C.I.)

$0.25(0.16,0.35)$

$0.27(0.17,0.38)$

$0.29(0.20,0.40)$

$0.21(0.12,0.32)$

$0.50(0.42,0.59)$

$0.27(0.18,0.37)$

$0.83(0.76,0.88)$

$0.44(0.41,0.46)$

$0.09(0.09,0.10)$

$0.46(0.36,0.56)$

$0.55(0.53,0.57)$

$0.44(0.41,0.47)$

$0.11(0.03,0.22)$

$0.32(0.25,0.39)$

$0.37(0.33,0.42)$

$0.15(0.06,0.25)$

$0.07(0.05,0.10)$

$0.32(0.21,0.44) 3851 / 1229$

Ev/N

$19 / 71$

$23 / 86$

$442 / 4692$

$1458 / 2667$

$481 / 1103$

$49 / 155$

$165 / 44$

$8 / 55$

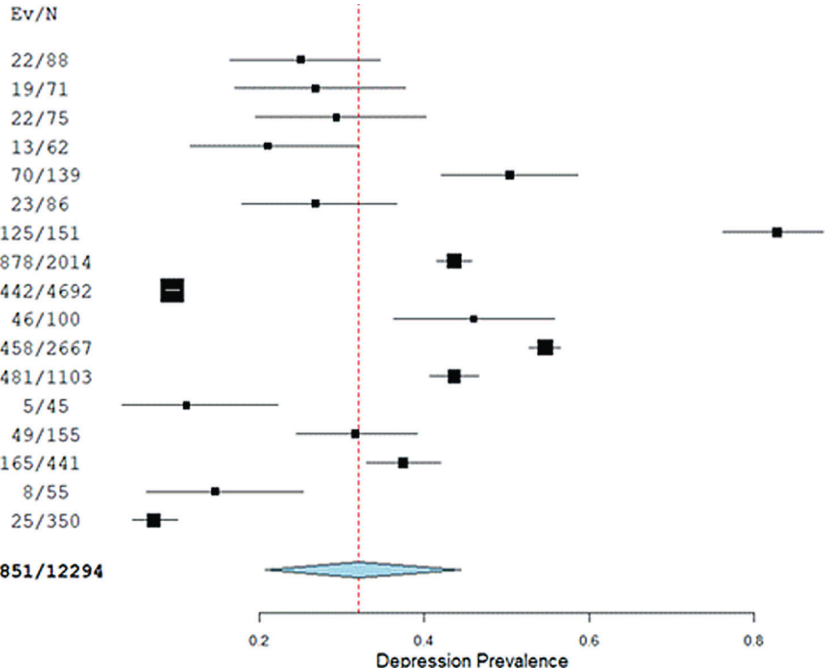

Figure 4. Pooled prevalence of depression among nurses $(\mathrm{Q}=2659.04, \mathrm{df}=16)$. 
pooled prevalence were found with respect to the degree of depression; mild (23.8\%), moderate (11\%), severe (4\%) and highly severe (1.7\%). While analysing subgroups; females had a significantly $(P=0.001)$ higher prevalence of depression $(27.9 \%)$ than males $(22.5 \%)$ but there were no significant differences $(P=0.09)$ in the prevalence rate between those who were married (26.9\%) and single (28.4\%) (Table 2 ). The pooled prevalence of depression $(61.2 \%, 95 \% \mathrm{CI}=16.9 \%-96.2 \%)$ from the Eastern Mediterranean region $\left(P<0.00, I^{2}=99\right)$ reported a higher prevalence than in Europe (30.9\%, 95\% CI=20.4\%-42.5\%) and combined prevalence from the Western Pacific and South-East Asian Regions (27.4\%, CI=13.0\%-44.7\%) (Table 3).

PTSD and insomnia: An imperceptible number of studies have evaluated PTSD which occurs as a secondary outcome due to stress (PTSD) $[13,32,48]$. The pooled prevalence of PTSD and insomnia at $I^{2}=96.43(P<0.001)$ and $I^{2}=98.56(P<0.001)$ was $18.6 \%(95 \% \mathrm{CI}=4.8 \%-38.3 \%)$ and $38.3 \%(95 \% \mathrm{CI}=5.8 \%-78.6 \%)$ respectively (Table 2).

Risk factors for mental ailments: The significant risk factors for anxiety, stress and depression as reported in different studies [9,11-13,29,34,36,37,39,40,42-48] were; direct care of COVID-19 patients, being a female, having low self-efficacy and resilience, poor social support and having physical symptoms (sore-throat, breathlessness, cough, lethargy, myalgia, fever). However specific risk factors for each of the mental outcomes evaluated from studies are as follows: i) anxiety: married women, having a child, aged 31-40 years, working in critical sectors, lack of access to personal protective equipment (PPE) and suspected infection; ii) stress: high workload, quarantine and impaired work ability; and iii) depression: being single, aged 21-30 years, having one child, having no support from family and hospital authority, being current smokers, having a chronic illness, poor sleep quality, high anxiety and redeployment outside professional boundaries. Moreover, explicit scrutiny was undertaken to identify the role of workplace on specific mental health outcomes. Anxiety, stress and depression were more common in nurses working in COVID-19 wards, COVID-19 designated hospital and critical sectors (Intensive Care Unit-ICU, surgery, emergency). However, a single study has reported that nurses working in normal wards were having more anxiety than those in ICU. A plausible reason attributed by the respective authors is that the safety environment and personal protection procedures in ICU enhanced the confidence of the nurses [42]. (Table 1).

\section{Publication bias and sensitivity analysis}

We carried out an Egger's publication bias plot [27] to detect the presence of publication bias which had an insignificant $P$ value for all the primary mental health outcomes; anxiety $(t=0.23, P=0.13)$, stress $(t=0.68$, $P=0.52)$, depression $(t=0.52, P=0.61)$, PTSD $(t=0.08, P=0.47)$ indicating no substantial publication bias. In addition, a visual inspection of funnel plots for a Logit event rate of prevalence's for mental health outcomes against corresponding standard error suggests evidence for the absence of publication bias (Figure S1, S2 and S3 in the Online Supplementary Document). However, the publication bias was not addressed for the mental health outcome-insomnia, since there were only two studies.

A leave-one-out sensitivity analysis was performed for all the studies included under each of the specified mental health outcomes; anxiety $(n=21)$, depression $(n=17)$, stress $(n=10)$, PTSD $(n=3)$, insomnia $(n=2)$. Obviously no individual study had a considerable influence on the overall pooled prevalence for different mental health outcomes. Furthermore, the overall prevalence as reported in sensitivity analysis were; anxiety-33.2\% (95\% CI $=24.0 \%-43.0 \%$ ), stress-40.4\% (95\% CI $=25.2 \%-56.6 \%)$, depression-32.6\% (95\% CI $=21.0 \%-45.5 \%$ ), PTSD-19\% (95\% CI=5.0\%-38.0\%), insomnia-38.4\% (95\% CI=6.0\%-78.6\%).

\section{DISCUSSION}

This systematic review and meta-analysis assessed the prevalence of mental health outcomes among nurses. Comparatively, higher rates of poor mental health outcomes namely, anxiety, stress, depression, PTSD and insomnia were observed among nurses from different parts of the world supporting our hypothesized statement. A high level of anxiety can be a precursor for other mental health outcomes, namely depression and insomnia [49] which has already been reported at moderate levels in our study. Depression bears the greatest burden of disability among mental and behavioural disorders [50]. This ultimately leads to many effects, including reduced job performance and quality, a greater risk of injuries at work, increased tardiness or absenteeism, impaired presenteeism, higher turnover rates, and a greater propensity for substance abuse [51-54]. It can be serious enough to lead to suicide in certain circumstances [55]. Studies conducted during the SARS outbreak also reported a similar proportion of depression, insomnia, emotional distress [56,57]. However, a lower prevalence rate was obtained for PTSD in our study as compared to a higher prevalence during SARS [56]. This 
discrepancy may be due to the inclusion of a significant number of studies conducted during the initial phase of COVID-19. Besides, this review highlighted the risk factors of mental health outcomes. There is no previous systematic review or meta-analysis that explored the prevalence of mental health outcomes among nurses during the COVID-19 pandemic.

Our study found that mental illness is common among nurses working in the frontline with COVID-19 patients. The prevalence of mental illness was not the same across different demographic groups and showed differences based on gender, marital status and geographic location. Multiple recent studies that explored the psychological status of health care professionals during the COVID-19 pandemic have emphasized the raised level of different types of pressures on nurses including higher exhaustion, higher stress perception, depressive symptoms, and lower job fulfilment compared to other health care professionals. This could be primarily due to the higher workload on them compared to their colleagues and the nature of their job responsibility that requires a prolonged duration of follow-up and direct contact with the patients.

Multiple variables were subjected to subgroup analysis to determine their role in the development of mental ailments. We did not find any statistically significant difference between subgroups based on gender or the marital status: males (27\%) vs females (33.3\%)) and (married (35.7\%) vs single (31.5\%). However, females had a significantly higher prevalence of depression (27.9\%) than males $(22.5 \%)$. At the same time, the prevalence of stress, PTSD and insomnia were not reported for subgroups in the studies included in the analysis. Our study identified the following risk factors for anxiety, stress and depression among nurses: caring for and being in direct contact with COVID-19 patients, being a female, low self-efficacy, resilience and social support and having physical symptoms (sore-throat, breathlessness, cough, lethargy, myalgia, fever). Comparatively, another study [58] reported that effective coping and self-adjustment strategies are essential for disaster rescuers to mitigate the burden of mental health problems; that they play a discerning role in responding efficiently and in a sustained and determined manner to the disaster. Furthermore, a similar study [59] have also reported that team cohesiveness is important to ensure that participants ask for support, alleviate pressure and develop coping strategies and mental resilience while responding to a new infectious disease outbreak. Similarly, lower self-efficacy, observed in our study as a risk factor for mental ailments is consistent with findings from earlier studies [60,61]. A recent report by the Department of Statistics in Canada has explored gender differences in mental health during the COVID-19 pandemic and reported that females are more likely to report worse mental health [62]. Another report from the United Kingdom has mentioned that the decline in the mental well-being of females was twice that for males. They emphasised the contribution of social factors in increasing the level of mental burden. Being socially active and having a larger social network before the pandemic was strongly associated with higher declines in the level of mental well-being after the pandemic. Females reported having more close friends before the pandemic than males, and higher loneliness than males after the pandemic [63]. Besides being a worker, woman having a family and other responsibility such as children were other important contributors to the negative impact on the mental well-being of females [64]. As seen in younger nurses in the study, an increased risk of developing psychological morbidity was also reported during the SARS outbreak [12]. This may be attributed to the insufficient expertise and inadequate preparation of younger nurses. However, the risk of anxiety was more pronounced in middle-aged nurses, which may be due to their perception of the family being getting infected by them. There was no significant difference between the nurses who were married and single in the prevalence of depression. On the contrary, several studies have reported that married nurses are having more serious depression than those who were single [65-67]. The inclusion of nurses from various parts of the world with cultural and geographical differences may be given as a potential reason for this.

Prevalence of mental illnesses was more common among nurses from the Eastern Mediterranean region compared to Europe and the combined prevalence reported from Western Pacific, South-East Asian region. A previous report by the United Nations highlighted that there is insufficient primary care in many countries in the Middle East region and that the health care system is fragmented. Besides, many factors such as unemployment, poverty, inadequate social safety nets, insufficiently responsive institutions and governance systems, and the economic shrinkage deeply impact the psychological and social status, and the general well-being of these populations [68]. Different studies from different regions have examined the negative impact of the COVID-19 pandemic on the relationship of different society segments. They have highlighted the negative influence on the relationship and communications between the members of the same family, colleagues at work and society as a whole [69-71].

High workload; cited as a contributing factor for negative mental health outcomes in most of the studies is intrinsically related to the global scarcity of nurses. The global shortage of nurses is 5.9 million, with the largest shortfall coming from low middle-income countries such as Pakistan, Nigeria, Indonesia and India. This is 
further substantiated by high stress reported from the South-East Asian region (47.2\%) and the Eastern Mediterranean region (61.6\%) in our review. To accelerate the response of the public health system during the pandemic as well as to attain sustainable development goals, there should be an increased investment for the nursing workforce in the subsequent years. An outlay of proximate US $\$ 10$ per capita in low and lower-middle-income countries is required to augment the already existing nursing education [2].

\section{Strengths and limitations}

Our systematic review and meta-analysis have many strengths:

1. To the best of our knowledge, this is the first systematic review and meta-analysis that has addressed the global prevalence of mental health outcomes among nurses during the COVID-19;

2. Data abstraction and quality assessment performed by two independent investigators which increases the robustness of our findings;

3. Freeman Tukey Double Arcsine transformation was employed to get pooled prevalence;

4. Included studies yielded data on nurses from different regions of the world which increase the generalisability of our estimates;

5. Sensitivity and subgroup analysis were done to explore the robustness of our estimates.

Nevertheless, our systematic review and meta-analysis have limitations:

1. Due to the lockdown policy implemented throughout the world, most of the included studies were cross-sectional web-based surveys, so that there could be a possibility of sampling bias;

2. The psychological status of nurses in the included studies was not evaluated before the pandemic. This restricted our ability to explore additional psychological burden on nurse due to the COVID-19 pandemic as we do not have data on their psychological status before it;

3. Substantial heterogeneity was identified among studies, which could be raised due to the difference in the assessment scales (variation in cut-offs scores) that were used across the studies to explore mental health outcomes;

4. Six studies were excluded as the authors of respective papers were not reachable;

5. Only research papers published in English were included, contributing to the lack of some studies from Asian countries in particular.

\section{CONCLUSION AND RECOMMENDATIONS}

The current systematic review and meta-analysis have contributed empirical evidence on the deleterious effect of COVID-19 on the psychological health of nurses and in particular, manifested as anxiety, stress, depression, PTSD and insomnia. Consequently, health care organizations should prioritize the needs of nurses by providing various provisions such as short duty and adequate rest hours, sufficient protective supplies, online support services and due recognition to mitigate the vulnerability for poor mental health outcomes. The disaster preparedness plan envisaged by nations should have provisions to address the mental health of nurses which includes; regular screening for mental health issues, physical symptoms, promotion of coping strategies and resilience, targeted interventions to prevent PTSD. Furthermore, greater investment in addressing the global shortage of nurses should be given priority in national health policies especially in lower-middle-income countries that will make a substantial contribution to reacting to potential pandemics through decreased mental health demands on nurses. Moreover, attractive salary packages should be offered for preventing the emigration of nurses from lower-middle-income countries.

Our systematic review and meta-analysis suggest interventions to improve the psychological well-being of nurses during the COVID-19 pandemic. Counselling support should be provided through online workshops to enable nurses and other health care providers to cope with any potential psychological problems. Manpower should be increased and better resources allocation is recommended. Rotating nurses, providing flexible working schedules and encouraging nurses to use psychological support services are highly recommended. Further, studies evaluating the efficacy of mental health services to reduce the occurrence of poor mental health outcomes among nurses need to be carried out to ramp up the existing services. 


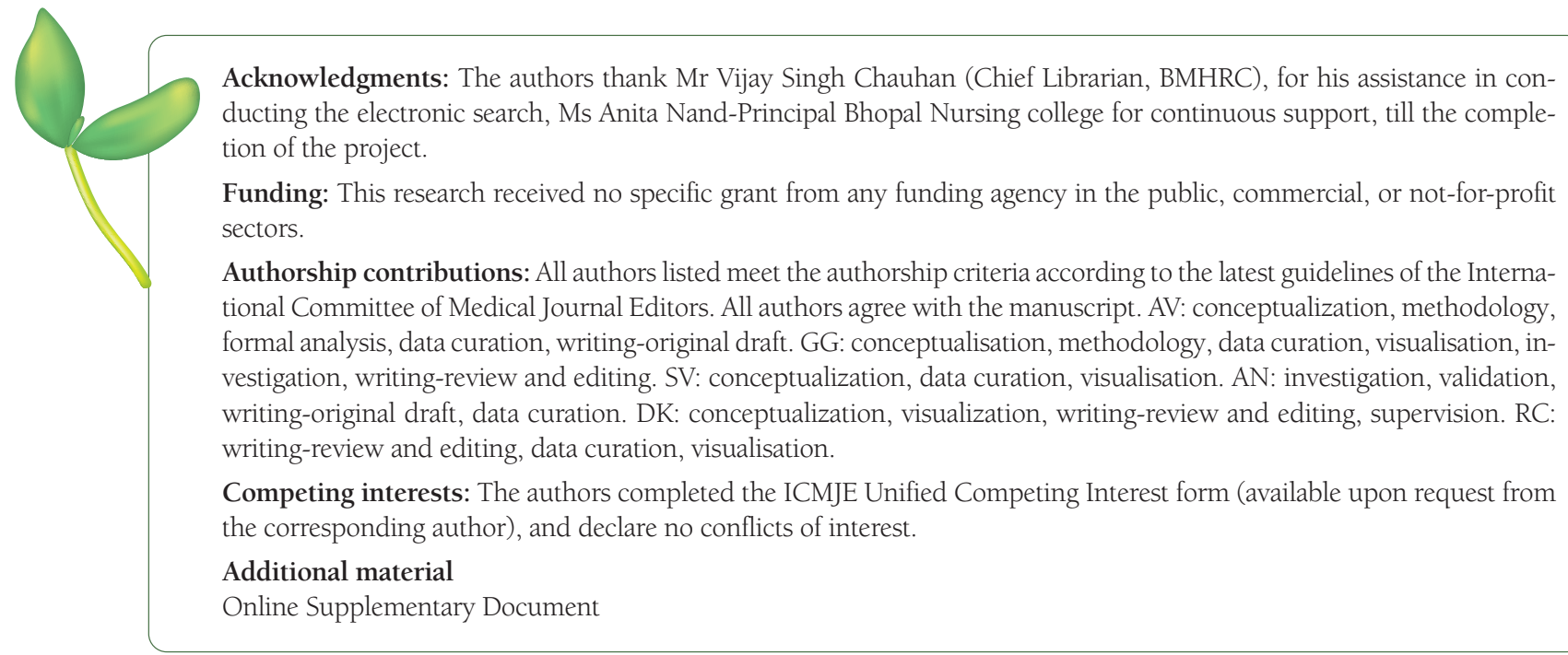

1 World Health Organization. Weekly epidemiological update - 17 November 2020. Available: https://www.who.int/publications/m/item/weekly-epidemiological-update_-17-november-2020. Accessed: 1 January 2021.

2 World Health Organization. State of the World's Nursing Report - 2020. Available: https://www.who.int/publications/i/ item/9789240003279. Accessed:9 January 2021.

3 Chen CS, Wu HY, Yang P, Yen CF. Psychological distress of nurses in Taiwan who worked during the outbreak of SARS. Psychiatr Serv. 2005;56:76-9. Medline:15637196 doi:10.1176/appi.ps.56.1.76

4 Chan SSC, Leung GM, Tiwari AFY, Salili F, Leung SSK, Wong DCN, et al. The impact of work-related risk on nurses during the SARS outbreak in Hong Kong. Fam Community Health. 2005;28:274-87. Medline:15958885 doi:10.1097/00003727200507000-00008

5 Bukhari EE, Temsah MH, Aleyadhy AA, Alrabiaa AA, Alhboob AA, Jamal AA, et al. Middle east respiratory syndrome coronavirus (MERS-CoV) outbreak perceptions of risk and stress evaluation in nurses. J Infect Dev Ctries. 2016;10:845-50. Medline:27580330 doi:10.3855/jidc.6925

6 Ji D, Ji YJ, Duan XZ, Li WG, Sun ZQ, Song XA, et al. Prevalence of psychological symptoms among Ebola survivors and healthcare workers during the 2014-2015 Ebola outbreak in Sierra Leone: A cross-sectional study. Oncotarget. 2017;8:12784-91. Medline:28061463 doi:10.18632/oncotarget.14498

7 Maunder RG, Lancee WJ, Rourke S, Hunter JJ, Goldbloom D, Balderson K, et al. Factors associated with the psychological impact of severe acute respiratory syndrome on nurses and other hospital workers in Toronto. Psychosom Med. 2004;••••:93842. Medline:15564361 doi:10.1097/01.psy.0000145673.84698.18

8 Poon E, Liu K, Cheong D, Lee C, Yam L, Tang W. Impact of severe acute respiratory syndrome on anxiety levels of frontline health care workers. Hong Kong Med J. 2004;10:325-30. Medline:15479961

9 Dal'Bosco EB, Floriano LSM, Skupien SV, Arcaro G, Martins AR, Anselmo ACC. Mental health of nursing in coping with COVID-19 at a regional university hospital. Rev Bras Enferm. 2020;73:e20200434. Medline:32667576 doi:10.1590/00347167-2020-0434

10 Salopek-Žiha D, Hlavati M, Gvozdanovi Z, Gaši M, Placento H, Jaki H, et al. Differences in distress and coping with the covid-19 stressor in nurses and physicians. Psychiatr Danub. 2020;32:287-93. Medline:32796800 doi:10.24869/psyd.2020.287

11 Shahrour G, Dardas LA. Acute stress disorder, coping self-efficacy and subsequent psychological distress among nurses amid COVID-19. J Nurs Manag. 2020;28:1686-95. Medline:32767827 doi:10.1111/jonm.13124

12 Hong S, Ai M, Xu X, Wang W, Chen J, Zhang Q, et al. Immediate psychological impact on nurses working at 42 government-designated hospitals during COVID-19 outbreak in China: A cross-sectional study. Nurs Outlook. 2021;69:6-12. Medline:32919788 doi:10.1016/j.outlook.2020.07.007

13 Wang YX, Guo HT, Du XW, Song W, Lu C, Hao WN. Factors associated with post-traumatic stress disorder of nurses exposed to corona virus disease 2019 in China. Medicine (Baltimore). 2020;99:e20965. Medline:32590808 doi:10.1097/ MD.0000000000020965

14 Lai J, Ma S, Wang Y, Cai Z, Hu J, Wei N, et al. Factors Associated With Mental Health Outcomes Among Health Care Workers Exposed to Coronavirus Disease 2019. JAMA Netw Open. 2020;3:e203976. Medline:32202646 doi:10.1001/jamanetworkopen.2020.3976

15 Ayanian JZ. Mental Health Needs of Health Care Workers Providing Frontline COVID-19 Care. JAMA Health Forum and COVID-19. JAMA. 2020;324:1155-6. Medline:32960258 doi:10.1001/jama.2020.18432

16 World Health Organization. Mental health and psychosocial considerations during the Covid-19 outbreak. Available: https:// www.who.int/docs/default-source/coronaviruse/mental-health-considerations.pdf. Accessed: 1 January 2021. 
17 Brooks SK, Dunn R, Amlôt R, Rubin GJ, Greenberg NA. Systematic, Thematic Review of Social and Occupational Factors Associated with Psychological Outcomes in Healthcare Employees during an Infectious Disease Outbreak. J Occup Environ Med. 2018;60:248-57. Medline:29252922 doi:10.1097/JOM.0000000000001235

18 World Health Organization. Rapid reviews to strengthen health policy and systems: a practical guide. 2018. Available: http:// www.who.int/alliance-hpsr/resources/publications/rapid-review-guide/en/. Accessed: 1 January 2021.

19 Liberati A, Altman DG, Tetzlaff J, Mulrow C, Gøtzsche PC, Ioannidis JPA, et al. The PRISMA statement for reporting systematic reviews and meta-analyses of studies that evaluate healthcare interventions: explanation and elaboration. BMJ. 2009;339:b2700. Medline:19622552 doi:10.1136/bmj.b2700

20 Ouzzani M, Hammady H, Fedorowicz Z, Elmagarmid A. Rayyan-a web and mobile app for systematic reviews. Syst Rev. 2016;5:210. Medline:27919275 doi:10.1186/s13643-016-0384-4

21 Loney PL, Chambers LW, Bennett KJ, Roberts JG, Stratford PW. Critical Appraisal of the Health Research Literature: Prevalence or Incidence of a Health Problem. Chronic Dis Can. 1998;19:170-6. Medline:10029513

22 Wallace BC, Dahabreh IJ, Trikalinos TA, Lau J, Trow P, Schmid CH. Closing the gap between methodologists and end-users: R as a computational back-end. J Stat Softw. 2012;49:1-15. doi:10.18637/jss.v049.i05

23 DerSimonian R, Laird N. Meta-analysis in clinical trials. Control Clin Trials. 1986;7:177-88. Medline:3802833 doi:10.1016/01972456(86)90046-2

24 Barendregt JJ, Doi SA, Lee YY, Norman RE, Vos T. Meta-analysis of prevalence. J Epidemiol Community Health. 2013;67:9748. Medline:23963506 doi:10.1136/jech-2013-203104

25 Higgins JPT, Thompson SG, Deeks JJ, Altman DG. Measuring inconsistency in meta-analyses. BMJ. 2003;327:557-60. Medline:12958120 doi:10.1136/bmj.327.7414.557

26 Higgins JPT, Thomas J, Chandler J, Cumpston M, Li T, Page MJ, et al. Cochrane Handbook for Systematic Reviews of Interventions version 6.2. Available: www.training.cochrane.org/handbook. Accessed: 1 January 2021.

27 Egger M, Smith GD, Schneider M, Minder C. Bias in meta-analysis detected by a simple, graphical test. BMJ. 1997;315:62934. Medline:9310563 doi:10.1136/bmj.315.7109.629

28 Skoda EM, Teufel M, Stang A, Jöckel KH, Junne F, Weismüller B, et al. Psychological burden of healthcare professionals in Germany during the acute phase of the COVID-19 pandemic: Differences and similarities in the international context. J Public Health (Oxf). 2020;42:688-95. Medline:32766787 doi:10.1093/pubmed/fdaal24

29 Zerbini G, Ebigbo A, Reicherts P, Kunz M, Messman H. Psychosocial burden of healthcare professionals in times of covid-19 - a survey conducted at the university hospital augsburg. Ger Med Sci. 2020;18:Doc05. Medline:32595421

30 Szepietowski JC, Krajewski P, Biłynicki-Birula R, Poznański P, Krajewska M, Rymaszewska J, et al. Mental health status of health care workers during the COVID-19 outbreak in Poland: One region, two different settings. Dermatol Ther. 2020;33:e13855. Medline:32779845 doi:10.1111/dth.13855

31 Bachilo EV, Barylnik JB, Shuldyakov AA, Efremov AA, Novikov DE. Mental health of medical workers during the COVID-19 pandemic in Russia: Results of a cross-sectional study. medRxiv. 2020. doi:10.1101/2020.07.27.20162610

32 Giusti EM, Pedroli E, D’Aniello GE, Stramba Badiale C, Pietrabissa G, Manna C, et al. The Psychological Impact of the COVID-19 Outbreak on Health Professionals: A Cross-Sectional Study. Front Psychol. 2020;11:1684. Medline:32754102 doi:10.3389/ fpsyg.2020.01684

33 Naser AY, Dahmash EZ, Al-Rousan R, Alwafi H, Alrawashdeh HM, Ghoul I, et al. Mental health status of the general population, healthcare professionals, and university students during 2019 coronavirus disease outbreak in Jordan: A cross-sectional study. Brain Behav. 2020;10:e01730. Medline:32578943 doi:10.1002/brb3.1730

34 Hu D, Kong Y, Li W, Han Q, Zhang X, Zhu LX, et al. Frontline nurses' burnout, anxiety, depression, and fear statuses and their associated factors during the COVID-19 outbreak in Wuhan, China: A large-scale cross-sectional study. EClinicalMedicine. 2020;24:100424. Medline:32766539 doi:10.1016/j.eclinm.2020.100424

35 Chen H, Sun L, Du Z, Zhao L, Wang L. A cross-sectional study of mental health status and self-psychological adjustment in nurses who supported Wuhan for fighting against the COVID-19. J Clin Nurs. 2020;29:4161-70. Medline:32757428 doi:10.1111/jocn.15444

36 Zhou Y, Yang Y, Shi T, Song Y, Zhou Y, Zhang Z, et al. Prevalence and Demographic Correlates of Poor Sleep Quality Among Frontline Health Professionals in Liaoning Province, China During the COVID-19 Outbreak. Front Psychiatry. 2020;11:520. Medline:32595534 doi:10.3389/fpsyt.2020.00520

$37 \mathrm{Tu}$ ZH, He JW, Zhou N. Sleep quality and mood symptoms in conscripted frontline nurse in Wuhan, China during COVID-19 outbreak: A cross-sectional study. Medicine (Baltimore). 2020;99:e20769. Medline:32590755 doi:10.1097/ MD.0000000000020769

38 Zhan YX, Zhao SY, Yuan J, Liu H, Gui LL, Zheng H, et al. Prevalence and Influencing Factors on Fatigue of First-line Nurses Combating with COVID-19 in China: A Descriptive Cross-Sectional Study. Curr Med Sci. 2020;40:625-35. Medline:32767264 doi:10.1007/s11596-020-2226-9

39 An Y, Yang Y, Wang A, Li Y, Zhang Q, Cheung T, et al. Prevalence of depression and its impact on quality of life among frontline nurses in emergency departments during the COVID-19 outbreak. J Affect Disord. 2020;276:312-5. Medline:32871661 doi:10.1016/j.jad.2020.06.047

40 Li R, Chen Y, Lv J, Liu L, Zong S, Li H, et al. Anxiety and related factors in frontline clinical nurses fighting COVID-19 in Wuhan. Medicine (Baltimore). 2020;99:e21413. Medline:32791757 doi:10.1097/MD.0000000000021413 
41 Do Duy C, Nong VM, Ngo Van A, Doan Thu T, Do Thu N, Nguyen Quang T. COVID-19-related stigma and its association with mental health of health-care workers after quarantine in Vietnam. Psychiatry Clin Neurosci. 2020;74:566-8. Medline:32779787 doi:10.1111/pcn.13120

42 Saricam M. COVID-19-Related anxiety in nurses working on front lines in Turkey. Nurs Midwifery Stud. 2020;9:178-81. doi:10.4103/nms.nms_40_20

43 Lee MCC, Thampi S, Chan HP, Khoo D, Chin BZB, Foo DPX, et al. Psychological distress during the COVID-19 pandemic amongst anaesthesiologists and nurses. Br J Anaesth. 2020;125:e384-6. Medline:32792139 doi:10.1016/j.bja.2020.07.005

44 Labrague LJ, de los Santos JA. COVID-19 anxiety among frontline nurses: Predictive role of organisational support, personal resilience and social support. J Nurs Manag. 2020;28:1653-61. Medline:32770780 doi:10.1111/jonm.13121

45 Badahdah A, Khamis F, Al Mahyijari N, Al Balushi M, Al Hatmi H, Al Salmi I, et al. The mental health of health care workers in Oman during the COVID-19 pandemic. Int J Soc Psychiatry. 2020. Online ahead of print. Medline:32635837 doi:10.1177/0020764020939596

46 Pouralizadeh M, Bostani Z, Maroufizadeh S, Ghanbari A, Khoshbakht M, Alavi SA, et al. Anxiety and depression and the related factors in nurses of Guilan University of Medical Sciences hospitals during COVID-19: A web-based cross-sectional study. Int J Afr Nurs Sci. 2020;13:100233. Medline:32837911

47 Wilson W, Raj JP, Rao S, Ghiya M, Nedungalaparambil NM, Mundra H, et al. Prevalence and Predictors of Stress, anxiety, and Depression among Healthcare Workers Managing COVID-19 Pandemic in India: A Nationwide Observational Study. Indian J Psychol Med. 2020;42:353-8. Medline:33398224 doi:10.1177/0253717620933992

48 Chew NWS, Lee GKH, Tan BYQ, Jing M, Goh Y, Ngiam NJH, et al. A multinational, multicentre study on the psychological outcomes and associated physical symptoms amongst healthcare workers during COVID-19 outbreak. Brain Behav Immun. 2020;88:559-65. Medline:32330593 doi:10.1016/j.bbi.2020.04.049

49 Teles MAB, Barbosa MR, Vargas AMD, Gomes VE, Ferreira EF, Martins AME de BL, et al. Psychosocial work conditions and quality of life among primary health care employees: a cross sectional study. Health Qual Life Outcomes. 2014;12:72. Medline:24884707 doi:10.1186/1477-7525-12-72

50 World Health Organization. Depression. Available: https://www.who.int/news-room/fact-sheets/detail/depression. Accessed: 25 February 2021.

51 Brandford AA, Reed DB. Depression in registered nurses: A state of the science. Workplace Health Saf. 2016;64:488-511. Medline:30209987 doi:10.1177/2165079916653415

52 Stansfeld S, Feeney A, Head J, Canner R, North F, Marmot M. Sickness absence for psychiatric illness: The Whitehall II study. Soc Sci Med. 1995;40:189-97. Medline:7899931 doi:10.1016/0277-9536(94)E0064-Y

53 Junqueira MA de B. Santos MA dos, Araújo LB de, Ferreira MC de M, Giuliani CD, Pillon SC. Depressive symptoms and drug use among nursing staff professionals. Esc Anna Nery. 2018;22:e20180129. doi:10.1590/2177-9465-ean-2018-0129

54 Woo JM, Kim W, Hwang TY, Frick KD, Choi BH, Seo YJ, et al. Impact of depression on work productivity and its improvement after outpatient treatment with antidepressants. Value Health. 2011;14:475-82. Medline:21669372 doi:10.1016/j. jval.2010.11.006

55 Silva DSD, Tavares NVS, Alexandre ARG, Antunes Freitas DA, Brêda MZ, Cícera dos Santos M, et al. Depression and risk of suicide in professional Nursing: integrative review. Rev Esc Enferm. 2015;49:1023-31. doi:10.1590/S0080-623420150000600020

56 Su TP, Lien TC, Yang CY, Su YL, Wang JH, Tsai SL, et al. Prevalence of psychiatric morbidity and psychological adaptation of the nurses in a structured SARS caring unit during outbreak: A prospective and periodic assessment study in Taiwan. J Psychiatr Res. 2007:41:119-30. Medline:16460760 doi:10.1016/j.jpsychires.2005.12.006

57 Nickell LA, Crighton EJ, Tracy CS, Al-Enazy H, Bolaji Y, Hanjrah S, et al. Psychosocial effects of SARS on hospital staff: Survey of a large tertiary care institution. CMAJ. 2004;170:793-8. Medline:14993174 doi:10.1503/cmaj.1031077

58 van der Velden PG, van Loon P, Benight CC, Eckhardt T. Mental health problems among search and rescue workers deployed in the Haiti earthquake 2010: A pre-post comparison. Psychiatry Res. 2012;198:100-5. Medline:22469424 doi:10.1016/j. psychres.2012.02.017

59 McAlonan GM, Lee AM, Cheung V, Cheung C, Tsang KWT, Sham PC, et al. Immediate and sustained psychological impact of an emerging infectious disease outbreak on health care workers. Can J Psychiatry. 2007;52:241-7. Medline:17500305 doi:10.1177/070674370705200406

60 Wahlberg L, Nirenberg A, Capezuti E. Distress and coping self-efficacy in inpatient oncology nurses. Oncol Nurs Forum. 2016;43:738-46. Medline:27768125 doi:10.1188/16.ONF.738-746

61 Chang Y, Wang PC, Li HH, Liu YC. Relations among depression, self-efficacy and optimism in a sample of nurses in Taiwan. J Nurs Manag. 2011;19:769-76. Medline:21899630 doi:10.1111/j.1365-2834.2010.01180.x

62 Moyser M. Gender differences in mental health during the COVID-19 pandemic, Available: https://www150.statcan.gc.ca/nl/ pub/45-28-0001/2020001/article/00047-eng.htm. Acessed:25 February 2021

63 Etheridge B, Spantig L. The Gender Gap in Mental Well-Being During the Covid-19 Outbreak: evidence from the UK. Available: www.iser.essex.ac.uk. Accessed:25 February 2021

64 Centre for Addiction and Mental Health. COVID-19 pandemic adversely affecting mental health of women and people with children. Available: https://www.camh.ca/en/camh-news-and-stories/covid-19-pandemic-adversely-affecting-mental-health-ofwomen-and-people-with-children. Accessed:25 February 2021.

65 Halvani GH, Salmani Nodoushan I, Hoboubati H, Salmani Nodoushan M, Jafari Nodoushan R, Hajian N. Effect of Shift Work on the Frequency of Depression in Nursing Staff of Yazd University of Medical Sciences. J Comm Health Res. 2012;1:104-9. 
66 Ohida T, Kamal A, Sone T, Ishii T, Uchiyama M, Minowa M, et al. Night-Shift Work Related Problems in Young Female Nurses in Japan. J Occup Health. 2001;43:150-6. doi:10.1539/joh.43.150

67 Ardekani ZZ, Kakooei H, Ayattollahi SM, Choobineh A, Seraji GN. Prevalence of mental disorders among shift work hospital nurses in Shiraz, Iran. Pak J Biol Sci. 2008;11:1605-9. Medline:18819649 doi:10.3923/pjbs.2008.1605.1609

68 United Nations. The Impact of COVID-19 on the Arab Region An Opportunity to Build Back Better. Available: www.unocha. org/sites/unocha/files/Global-Humanitarian-Response-Plan-COVID-19.pdf. Accessed:1 January 202:

69 Karaye IM, Horney JA. The Impact of Social Vulnerability on COVID-19 in the U.S.: An Analysis of Spatially Varying Relationships. Am J Prev Med. 2020;59:317-25. Medline:32703701 doi:10.1016/j.amepre.2020.06.006

70 Banerjee D, Rai M. Social isolation in Covid-19: The impact of loneliness. Int J Soc Psychiatry. 2020;66:525-7. Medline:32349580 doi:10.1177/0020764020922269

71 Naser AY, Al-Hadithi HT, Dahmash EZ, Alwafi H, Alwan SS, Abdullah ZA. The effect of the 2019 coronavirus disease outbreak on social relationships: A cross-sectional study in Jordan. Int J Soc Psychiatry. 2020. Online ahead of print. Medline:33103566 doi:10.1177/0020764020966631 